\title{
Health effects of breast feeding beyond traditional perspective- Literature review.
}

\author{
Amir Ali Samnani ${ }^{*}$, Mehak Samnani², Sohail Shaikh ${ }^{3}$, Naveed Bhutto ${ }^{3}$ \\ ${ }^{1}$ Provincial Nutrition Cell, Sindh, Karachi, Pakistan \\ ${ }^{2}$ Department of Health, Aga Khan University, Karachi, Pakistan \\ ${ }^{3}$ Nutrition Support Program, Karachi, Pakistan
}

\begin{abstract}
High levels of malnutrition have serious consequences on the health and survival of infants and young children. According to National Nutrition Survey (NNS-2011), almost 44\% of children in Pakistan are stunted, $30 \%$ are underweight and $15 \%$ are severely wasted. One of the major reasons for such high rates of malnutrition is inappropriate Infant and Young Child Feeding (IYCF) practices which includes breastfeeding and complementary feeding. The prevalence of exclusive breastfeeding was $20.9 \%$ at 4 months and $12.9 \%$ at 6 months of age. Similarly, although $51.3 \%$ mothers started giving semisolid foods to their children at the recommended 6-8 months of age, the proportion achieving minimum dietary diversity (children who received foods of 4 or more food groups) was only $3 \%$.
\end{abstract}

Keywords: Breastfeeding, Health effects, Malnutrition, Dietary, WHO.

Accepted on October $05^{\text {th }}, 2017$

\section{Introduction}

Various studies have linked malnutrition with inappropriate feeding practices. The World Health Organization (WHO) and United Nations International Children's Emergency Fund (UNICEF), with the broad participation of many stakeholders, developed the Global Strategy for IYCF to rejuvenate global commitment for improving infant and young child nutrition by promoting, protecting and supporting appropriate IYCF in general and breastfeeding in specific [1-3].

It is highly surprising that despite of common breastfeeding practice; exclusive breastfeeding remains uncommon in developing countries [4]. There is still a big challenge for healthcare professionals to encourage women to breastfeed exclusively. The World Health Organization (WHO) recommends exclusive breastfeeding in initial 6 months of life and continuing breastfeeding up to 2 years of age and beyond along with complementary feeding. Obviously, a focus both on initiating and continuing breastfeeding is very important to reduce the risk of failure on breastfeeding start and maintenance [4].

Despite, knowing the fact that breast milk is highly cost effective and has wide range of health implications, the prevalence of exclusive breastfeeding in low and middle- income countries among infants less than 6 months of age is only 37\% [5]. Breast feeding improves the health, nutrition, survival and overall cognitive and intellectual development of the children [6]. The benefits of breast feeding are not limited to the baby, but it also saves mother's life and provide a natural mean for spacing between successive child.

The scope of this writing was limited to the health effects of breastfeeding in children's. This Literature review aimed at identifying the long-term health benefits of breastfeeding among children's in later stages of their lives. This paper outlined that fact that breast feeding not only protects from infectious disease, but it also protects from the spectrum of adverse health outcomes beyond the traditional perspectives.

\section{Review of Related Literature}

The articles were selected using the Wiley online library database from "Acta Paedriatrica journal", using the search term "Breastfeeding and health benefits". Since all the selected study for this writing were based on systematic review and metaanalysis, systematic review and meta-analysis are considered as the best level of evidences. Findings of these studies were compiled after a pooled analysis from multiple studies, so they are well supported and connected with other available literature.

Sankar et al. [7] conducted a systematic review and metaanalysis to synthesize the effect of optimal breast feeding on allcause mortality and infection related infant and child mortality rates in children aged $0-23$ months. They found that, the risk of all-cause mortality when compared with exclusive breastfed infants of age 0-5 months; had three-fold higher in partially breastfed infants and 14 folds higher in non-breastfed infants. When comparing the infection related mortality in exclusive breastfed infants of age $0-5$ months, the risk was 4.6 and 8.7 times higher in partially and non-breastfed infants respectively. They further added that even partial breastfeeding had modest protective effect compared to no breastfeeding [7].

Similarly, Horta et al. [8] examined the evidence on the association between breastfeeding and overweight/obesity, blood pressure, total cholesterol and type 2 diabetes by conducting a systematic review. They found that, breastfed subjects were $13 \%$ less likely to be classified as obese/overweight. The magnitude of protection is larger in adolescents and lesser in later stage of life. Likewise, it was also highlighted that there is an association between breastfeeding and prevalence of type 2 diabetes. However, no association of breastfeeding with blood pressure and total cholesterol was evident from this review [8]. 
Another study based on systematic review analysis explored the breastfeeding association with asthma and allergic aspect of health [9]. They found that, there is low grade quality evidence that longer duration of breastfeeding is associated with a reduced risk of asthma in children 5-18 years.

The mechanism that reduce asthma to greater extent is through respiratory infections in childhood. In addition to asthma, allergic rhinitis and eczema were also assessed. It was found that, there is very low to low grade quality evidence that breastfeeding is associated with a reduced risk of allergic rhinitis in children up to 5 years of age and eczema in children up to 2 years of age. The reduced risk associated with allergy attributed to the viral protection from breastfeeding rather than protection from allergic rhinitis. No association was observed between breastfeeding and food allergy [9].

Finally, Horta et al. [8] evaluated the evidence on the association between breastfeeding and performance in intelligence test through systematic review. Some interested findings were observed and found that, breastfeeding is positively associated with improved IQ in childhood and adolescence. The average gain in IQ among breastfed children were 3.44 points.

\section{Discussion}

The possible reason identified in this review was the provision of cognitively stimulating environment for their infants by mothers who breastfed their child. In addition to this; presence of long chain poly-unsaturated fatty acids, such as arachidonic acid and docosahexaenoic acid in mother's milk would better explain this mechanism. This association persist after adjustment for maternal IQ as confounder. Moreover, findings also revealed that breastfeeding impacts on schooling and earning potential in adulthood [10].

\section{Conclusion of the Study}

Thus, findings from this scientific writing has provided the broad array of health benefits of breastfeeding and provide strong justification to protect, promote and support breastfeeding, yet there is still much need to learn about the actual pathways and mechanisms of breastfeeding and breastmilk on complex physiological systems that include immune system, microbiome and brain development.

\section{References}

1. Ministry of Planning and Development, Ministry of National Health Services, Regulations and Coordination (2012). Pakistan national nutrition survey. 2011;36,45-49.

2. Mahmood H, Suleman Y, Hazir T, et al. Overview of the infant and young child feeding policy environment in Pakistan: Federal, Sindh and Punjab context. BMC Public Health. 2017;17(2):474.

3. http://www.who.int/nutrition/topics/global_strategy/en/

4. Suwantika AA, Postma MJ. Effect of breastfeeding promotion interventions on cost-effectiveness of rotavirus immunization in Indonesia. BMC Public Health. 2013;13(1):1106.

5. Victora CG, Bahl R, Barros AJ, et al. Breastfeeding in the $2^{1 \text { st }}$ century: Epidemiology, mechanisms, and lifelong effect. Lancet. 2016; 387(10017):475-490.

6. Rollins NC, Bhandari N, Hajeebhoy N, et al. Why invest, and what it will take to improve breastfeeding practices? Lancet. 2016;387(10017):491-504.

7. Sankar MJ, Sinha B, Chowdhury R, et al. Optimal breastfeeding practices and infant and child mortality: A systematic review and meta-analysis. Acta Paediatr. 2015;104(S467):3-13.

8. Horta BL, Loret De Mola C, Victora CG. Longterm consequences of breastfeeding on cholesterol, obesity, systolic blood pressure and type 2 diabetes: A systematic review and meta-analysis. Acta Paediatr. 2015;104(S467):30-37.

9. Lodge CJ, Tan DJ, Lau MXZ, et al. Breastfeeding and asthma and allergies: A systematic review and metaanalysis. Acta Paediatr. 2015;104(S467):38-53.

10. Horta BL, Loret De Mola C, Victora CG. Breastfeeding and intelligence: A systematic review and meta-analysis. ActaPaediatrica. 2015;104(S467):14-19.

\section{*Correspondence to:}

Amir Ali Samnani

Provincial Nutrition Cell

Sindh

Karachi

Pakistan

Tel: +92 21 4549525-28

E-mail:am_samnani@hotmail.com 